\title{
On the Cones of $\alpha$-and Generalized $\alpha$-Positivity for Quantum Field Theories with Indefinite Metric
}

\author{
By
}

\author{
Gerald HoFmANN *
}

\begin{abstract}
In order to construct a Krein-space theory (i.e., a $*$-algebra of (unbounded) operators which are defined on a common, dense, and invariant domain in a Krein space) the cones of $\alpha$-positivity and generalized $\alpha$-positivity are considered in tensor algebras. The relations between these cones, algebraic $\#$-cones, and involutive cones are investigated in detail.

Furthermore, an example of a $P$-functional $\phi$ defined on $\left(\mathbf{C}^{2}\right)_{\otimes}$ (tensor algebra over $\mathbf{C}^{2}$ ) not being $\alpha$-positive and yielding a non-trivial Krein-space theory is explicitely constructed. Thus, an affirmative answer to the question whether or not the method of $P$-functionals (introduced by Ôta) is more general than the one of $\alpha$-positivity (introduced by Jakóbczyk) is provided in the case of tensor algebras.
\end{abstract}

\section{§ 1. Introduction}

The present investigations are motivated by the algebraic approach to general (axiomatic) quantum field theory $(Q F T)$. The formalism introduced by Borchers [8] and Uhlmann [30] works for massive fields as well as for massless or gauge fields and is entirely equivalent to the Wightman axioms. Starting with a tensor algebra $E_{\otimes}$ over some nuclear space $E$ of test functions and a normalized continuous Hermitean functional $W \in E_{\otimes}{ }^{\prime}$ satisfying some further physically motivated conditions (linear program), a $Q F T$ is reconstructed by means of the GNS (Gelfand, Naimark, Segal) construction.

It is now necessary to distinguish between i) massive fields, and ii) massless or gauge fields. In the case of i) $W$ is taken to be a positive functional (Wightman functional), and thus the space of state vectors becomes a Hilbert space. In the case of ii) there are no-go theorems (see [22], [29]) stating that locality, covariance

Communicated by H. Araki, June 22, 1993. Revised October 13, 1993.

1991 Mathematics Subject Classifications : 46C20, 81T05

* Universität Leipzig, Fachbereich Mathematik und Informatik, Augustusplatz 10, D-04109 Leipzig, Germany 
and positivity cannot be satisfied at the same time. From the point of view of general QFT it seems to be better to keep covariance and locality, and thus the positivity condition has to be abandoned. Such models were discussed, e. g., in [2], $[7],[10],[14],[15]$. The GNS representation then gives a state space with indefinite metric. Hence, indefinite metric is a general feature of all local and covariant formulations of QED, but also of gauge theories of Yang-Mills type and other similar theories (see also [11], [21], [24]).

The pseudo-Wighman axioms introduced by Strocchi $[28],[23]$; $[6$; Chapter 10] are an axiomatic approach to such theories. A publication by Araki [3] considers the specific problem of group representation on a space with indefinite metric such as is found in the situation of Gupta-Bleuler QED, and this theory also applies to the algebraic approach to QFT with indefinite metric. Further discussions on this subject are contained in [9], [31].

The present note is concerned with one mathematical aspect of this formalism, namely the GNS representation in state spaces $\mathscr{K}$ with indefinite metric. In order of decreasing generality, $\mathscr{K}$ can be chosen to be :

a) a general space with indefinite inner product,

b) a Hilbert space with an inner product $\langle.,$.$\rangle ,$

connected to $(.$, . ) by a bounded linear Hermitean operator $G$ (Gram operator) such that $(.,)=.\langle.$, G. $\rangle$, $([5 ;$ p. 89] $)$.

c) a Krein space (see $\S 3$ ).

(Other choices of $\mathscr{K}$ are also posible.)

In order to make the theory mathematically manageable and in particular to avoid topological complications (e. g., in the case of c) $M^{\perp \perp} \subset \bar{M}$ holds for every subspace $M \subset \mathscr{K}$ and thus the results of [3] apply immediately), it is desirable to obtain a Krein space for $\mathscr{K}$ (see [4], [5], [16]). The pseudo-Wightman axioms are formulated in this case. There are also physically motivated conditions for achieving that goal (see $[20 ;$ Ch. 3$]$ ). Thus the following question arises in our context : Are there conditions upon the functional $\mathrm{W} \in \boldsymbol{E}_{\otimes}{ }^{\prime}$ so that the space of state vectors $\mathscr{K}$ becomes a Krein space. Answers were given by Yngvason [33], Jakóbczyk [17], Antoine and Ôta [1], [25], Morchio and Strocchi [23]. While topological methods were used in [33], [23], the considerations in $[1],[25],[17]$ are on a purely algberaic level.

For tensor algebras $E_{\otimes}$, Jakóbczyk introduced the concept of $\alpha$-positivity and showed that every $\alpha$-positve and invariant linear functional yields a Krein-space theory via GNS representation (see §3). Ôta generalized that to the case of arbitrary *-algebras with unity. Among others the concept of $\alpha$-positivity is generalized to the one of generalized $\alpha$-positivity (see $\S 3$ ). Let us also mention 
that there is a minor generalization of the concept of $\alpha$-positivity (see Remark 3.6), which coincides with the "usual" one in the interesting case of alg-\# cones in tensor algebras (Corollary 4. 6).

The aim of this note is threefold. 1) The setups introduced in [1], [25] are applied to the case of tensor algebras $E_{\otimes}$ (Proposition 3.1). 2) The cones derived from $\alpha$-positivity and generalized $\alpha$-positivity, respectively are discussed for $E_{\otimes}$. Along these lines it is shown that every cone of $\alpha$-positivity also is a cone of generalized $\alpha$-positivity (Proposition 4. 5). Furthermore it is investigated whether or not those cones are alg-\# cones and involutive cones, respectively (Proposition 4. 1). These considerations are of interest because the concepts of alg-\# cones and involutive cones fit tensor algebras very well, and all the results given in [13] apply. 3) About the relevance of the considerations given in $[1],[25]$ for the case of tensor algebras, the following question arises :

(Q) Are there P-functionals (see $\S 3$ ) on $E_{\otimes}$ which are not $\alpha$-positive and yield a Krein-space theory with indefinite metric via GNS representation?

Proposition 5.5 gives an affirmative answer to $(Q)$ and shows that for tensor algebras the results of $[1],[25]$ really apply to more functionals than those of [17].

From the mathematical point of view it is of interest to develop a general theory of unbounded representations of $*$-algebras, and there one has to deal with a class of representations which are not $*$-hermitean ([27]). Typical examples of such representations are $J$-representations on Krein spaces (see §3). The results obtained in this note fit this theory. Furthermore, in $\S 5$ there it is shown how to construct examples to test and discuss the general theory of unbounded representations.

The pattern of the present note is as follows. In $\S 2$ there are collected the definitions and facts from the theory of tensor algebras needed in the following. The GNS representation yielding a Krein space as the space of state vectors and the concepts related to it are recalled and applied to $E_{\otimes}$ in $\S 3$. The relations between cones of $\alpha$-positivity and generalized $\alpha$-positivity, alg-\# cones and involutive cones are discussed in detail in $\S 4$. The aim of $\S 5$ is to answer $(Q)$. There is explicitely constructed a functional $\Phi$ which yields a "non-trivial" Krein-space theory via GNS representation using the methods doveloped in $: 1],[25]$, while on the other hand the methods explained in [17], [18] do not apply to $\Phi$.

Physical interpretations of the theory reconstructed from $\Phi$ such as the construction of the gauge group, the distinction of the vacuum sector and the physical subspace of the space of virtual states will be discussed in a subsequent 
paper.

\section{§ 2. On Tensor Algebras and Alg-\# Cones}

Let us recall some definitions and facts of the theory of tensor algebras needed in the following. For the following let us be given a vector space $E$ over the field of complex numbers $\mathbf{C}$, and let

$$
E_{n}=E \otimes E \otimes \cdots \otimes E
$$

stand for the $n$-fold (algebraic) tensor product of $E$ by itself, $n \in \mathbf{N}$. The tensor algebra $E_{\otimes}$ over the basic space $E$ is then defined by

$$
E_{\otimes}=\mathbf{C} \oplus E_{1} \oplus E_{2} \oplus \cdots \quad \text { (direct sum), }
$$

i.e., the elements $f \in E_{\otimes}$ are of the form

$$
f=\left(f_{0}, f_{1}, \ldots, f_{N}, 0,0, \ldots\right)
$$

where $f_{n} \in E_{n}$, and all but finitely many $f_{n}=0, E_{0}=\mathbf{C}, E_{1}=E$. Further, $f_{n}$ will be called the $n$-th homogeneous component of $f$.

Defining algebraic operations componentwise by putting

$$
\begin{aligned}
(f+g)_{n} & =f_{n}+g_{n}, \\
(\mu f)_{n} & =\mu f_{n}, \\
(f g)_{n} & =\sum_{r+s=n} f_{r} \otimes g_{s},\left(f_{0} \otimes g_{n}=g_{n} \otimes f_{0}=f_{0} g_{n}\right)
\end{aligned}
$$

for $f, g \in E_{\otimes}, \mu \in \mathbf{C}(n=0,1,2, \ldots), E_{\otimes}$ becomes an (associative) algebra with unity $\mathbb{1}=(1,0,0, \ldots)$. If an involution “* $*$ is given on $E$, then $E_{\otimes}$ becomes a $*$ algebra by setting

$$
\begin{aligned}
& f^{*}=\left(f_{0}^{*}, f_{1}^{*}, \ldots, f_{N}^{*}, 0,0, \ldots\right), \\
& f_{n}^{*}=h^{(n) *} \otimes \cdots \otimes h^{(1) *}
\end{aligned}
$$

for $f_{n}=h^{(1)} \otimes \cdots \otimes h^{(n)} \in E_{n}(n \in \mathbf{N}), f_{0}^{*}=\bar{f}_{0}$, and using antilinearity of $*$.

Let us be given some $f=\left(0, \ldots, 0, f_{N_{1}}, \ldots, f_{N_{2}}, 0,0, \ldots\right) \in E_{\otimes}$, where $f_{N_{1}} \neq 0, f_{N_{2}}$ 
$\neq 0, N_{1}, N_{2} \in \mathbf{N}^{*}(:=\{0,1,2, \ldots\})$. Then put

$$
\begin{aligned}
& \operatorname{Grad}(f)=N_{2}, \operatorname{grad}(f)=N_{1} \text { for } f \neq \mathbf{0}, \\
& \operatorname{Grad}(\mathbf{0})=-\infty, \operatorname{grad}(\mathbf{0})=\infty,
\end{aligned}
$$

where $\mathbf{0}=(0,0, \ldots) \in \boldsymbol{E}_{\otimes}$.

For the following let $Q_{n} ; E_{\otimes} \longrightarrow\left(\bigoplus_{i=0}^{n} E_{i}\right)=: E^{n}$ denote the canonical projections, where $E^{n}$ is considered as a subspace of $E_{\otimes}$, i. e., for $f=\left(f_{0}, \ldots, f_{N}, 0\right.$, $0, \ldots) \in E_{\otimes}$ it follows

$$
Q_{n}(f)=\left(f_{0}, \ldots, f_{n}, 0,0, \ldots\right)
$$

(For further considerations and some applications of $Q_{n}$ the reader is refered to [12].)

Furthermore, let

$$
\check{f}_{n}=\left(0, \ldots, 0, f_{n}, 0,0, \ldots\right) \in \boldsymbol{E}_{\otimes},
$$

$f_{n} \in E_{n}$, and $\check{F}_{n}=\left\{\check{f}_{n} ; f_{n} \in F_{n}\right\}, F_{n} \subset E_{n}\left(n \in \mathbf{N}^{*}\right)$.

Let us now be given an operator $A: E_{\otimes} \longrightarrow E_{\otimes}$. Then, $A$ will be called graded, if $A\left(\check{E}_{m}\right) \subset \check{E}_{m}, m=0,1,2, \ldots$. In the case that $A$ is linear (resp. antilinear) and graded there are linear (resp. antilinear) operators $A_{n}: E_{n} \longrightarrow E_{n}(n=0,1,2, \ldots)$ such that

$$
A f=\left(A_{0} f_{0}, A_{1} f_{1}, \ldots\right)
$$

$f=\left(f_{0}, f_{1}, \ldots\right) \in E_{\otimes}$. In the following, $A=\left(A_{0}, A_{1}, \ldots\right)$ will be written. There is the following characterization of gradedness.

Lemma 2. 1. Let us be given a linear or antilinear operator $A$. Then $A$ is graded, if and only if

$$
A Q_{n}=Q_{n} A,
$$

$n=0,1,2, \ldots$, are satisfied.

Proof. $(\Leftarrow)$ : Let us be given $f=\left(f_{0}, \ldots, f_{N}, 0,0, \ldots\right) \in E_{\otimes}$. Then, $A \check{f}_{n} \in E^{n}(n$ $=0,1,2, \ldots)$ due to (1). Hence, for each $f_{n}$ there are $h_{i} \in E_{l}(i=0,1, \ldots, n)$ such that

$$
A \check{f}_{n}=\left(h_{0}, h_{1}, \ldots, h_{n}, 0,0, \ldots\right)
$$


Noticing

$$
\begin{aligned}
& Q_{n-1} A \breve{f}_{n}=\left(h_{0}, h_{1}, \ldots, h_{n-1}, 0,0, \ldots\right), \\
& A Q_{n-1} \check{f}_{n}=A(0)=0
\end{aligned}
$$

and using (1), $h_{j}=0 \quad(j=0,1, \ldots, n-1)$ follow. Hence $A \check{f}_{n} \in \check{E}_{n}$, and $A$ is graded.

$(\Rightarrow)$ : Assume that there is an $n_{0} \in \mathbf{N}^{*}$ such that $A Q_{n_{0}} \neq Q_{n_{0}} A$. So there is an $f=\left(f_{0}, \ldots, f_{N}, 0,0, \ldots\right) \in E_{\otimes}$ with $A Q_{n_{0}} f \neq Q_{n_{0}} A f, \operatorname{Grad}(f)=N$. i) Let $n_{0}>N$. Then, $A f \neq Q_{n_{0}} A f$. Hence $\operatorname{Grad}(A f)>n_{0}$, and $A$ is not graded. ii) Let $n_{0}<N$. Using the linearity (resp. antilinearity) of $A$,

$$
\begin{aligned}
A\left(f_{0}, \ldots, f_{n_{0}}, 0,0, \ldots\right) & \neq Q_{n_{0}} A\left(f_{0}, \ldots, f_{n_{0}}, 0,0, \ldots\right) \\
& +Q_{n_{0}} A\left(0, \ldots, 0, f_{n_{0}+1}, \ldots, f_{N}, 0,0, \ldots\right)
\end{aligned}
$$

and

$$
Q_{n_{0}} A\left(0, \ldots, 0, f_{n_{0}+1}, \ldots, f_{N}, 0,0, \ldots\right) \neq 0
$$

or

$$
\operatorname{Grad}\left(A\left(f_{0}, \ldots, f_{n_{0}}, 0,0, \ldots\right)\right)>n_{0}
$$

follow. (2) and $\left(2^{\prime}\right)$, respectively, imply that $A$ is not graded. The proof is completed.

Let us now define the class of alg-\# cones which fits the algebraic structure of $E_{\otimes}$ very well. Let us be given a subspace

$$
F=\bigoplus_{n=0}^{\infty} F_{n}
$$

of $E_{\otimes}$, where $F_{n} \subset E_{n}$. Further, let us consider an antilinear mapping \#: $E_{\otimes} \longrightarrow E_{\otimes}$ which satisfies

$$
\begin{gathered}
f^{\# \#}=f, \\
\left(Q_{n} f\right)^{\#}=Q_{n}\left(f^{\#}\right)
\end{gathered}
$$

for all $f \in E_{\otimes}, n=0,1,2, \ldots$. Notice that \# is bijective. Let us put 


$$
\{\boldsymbol{F}, \#\}=\left\{\sum_{i=1}^{M} f^{(i) \#} f^{(i)} ; f^{(\imath)} \in \boldsymbol{F}, \boldsymbol{M} \in \mathbf{N}\right\} .
$$

It is immediate that $\{F, \#\}$ is a convex cone (containing its apex 0 ). In the following such cones $\{F, \#\}$ will be called alg-\# cones. If \# additionally satisfies

$$
(f g)^{\#}=g^{\#} f^{\#}
$$

for all $f, g \in E_{\otimes}$, then $\{F, \#\}$ will be called involutive cone. Notice also that

$$
\boldsymbol{E}_{\otimes}^{+}=\left\{\boldsymbol{E}_{\otimes}, *\right\}=\left\{\sum_{i=1}^{M} f^{(i) *} f^{(i)} ; f^{(\imath)} \in \boldsymbol{E}_{\otimes}, \boldsymbol{M} \in \mathbf{N}\right\}
$$

is the well-known cone of positive elements of $E_{\otimes}$.

Further, a linear functional $T$ on $E_{\otimes}$ is called $\{F, \#\}$-positive if $T(k) \geq 0$ for all $k \in\{F, \#\}$. Among others, the $\{F, \#\}$-positivity of $T$ implies that the CauchySchwarz inequality

$$
\left|T\left(f^{\#} g\right)\right|^{2} \leq T\left(f^{\sharp} f\right) T\left(g^{\#} g\right),
$$

$f, g \in F$, is satisfied (see $\left[8\right.$; Lemma 2. 1]). Using $T=\left(T_{0}, T_{1}, \ldots\right)$, (3) and (6) imply

$$
\left|T_{n+m}\left(f_{n}^{\#} \otimes f_{m}\right)\right|^{2} \leq T_{2 n}\left(f_{n}^{\#} \otimes f_{n}\right) T_{2 m}\left(f_{m}^{\#} \otimes f_{m}\right), \quad f_{n}, f_{m} \in F, \quad n, m \in \mathbf{N}^{*} .
$$

In order to construct $\{F, \#\}$-positive linear functionals the following is of interest. Let us be given a linear functional $T$ and a sequence $\left(\omega_{n}\right)_{n=0}^{\infty}, \omega_{n}>0$, such that $\left(6^{\prime}\right)$ is satisfied in the modified version

$$
\left|T_{n+m}\left(f_{n}^{\#} \otimes f_{m}\right)\right|^{2} \leq \omega_{n} \omega_{m} T_{2 n}\left(f_{n}^{\#} \otimes f_{n}\right) T_{2 m}\left(f_{m}^{\#} \otimes f_{m}\right), T_{2 n}\left(f_{n}^{\#} \otimes f_{n}\right) \geq 0,
$$

$f=\left(f_{0}, f_{1}, \ldots\right) \in F$. Now the following question arises. Is it always possible to relate an $\{F, \#\}$-positive linear functional $S$ to $T$ ? With this in mind let us define the following.

Definition 2. 2. A sequence $\left(\beta_{n}\right)_{n=0}^{\infty}, \beta_{n} \geq 0$, is called to be of positive type (with respect to $T$ and $\{F, \#\}$ ), if the functional

$$
S=T^{(\beta)}:=\left(\beta_{0} T_{0}, \beta_{1} T_{1}, \beta_{2} T_{2}, \ldots\right)
$$

is $\{F, \#\}$-positive. 
The following example shows that $\left(6^{\prime \prime}\right)$ implies that there are always sequences of positive type.

Example 2. 3. Let us recursively define a sequence $\left(\beta_{m}\right)_{m=0}^{\infty}$ by $\beta_{2 n-1}=0, \beta_{0}=$ $\beta_{2}=2$,

$$
\begin{gathered}
\beta_{4 n}=\sum_{m=1}^{n} 2^{m} \omega_{2 n} \omega_{2 n-2 m}\left(\beta_{4 n-2 m}\right)^{2}+1 \\
\beta_{4 n+2}=\sum_{m=1}^{n} 2^{m} \omega_{2 n+1} \omega_{2(n-m)+1}\left(\beta_{2(2 n-m+1)}\right)^{2}+1,
\end{gathered}
$$

$n=1,2, \ldots$

Lemma 2. 4. The sequence $\left(\beta_{n}\right)_{n=0}^{\infty}$ is of positive type.

Proof. Setting $a_{n m}=T_{n+m}\left(f_{n}^{\#} \otimes f_{m}\right), f=\left(f_{0}, f_{1}, \ldots\right) \in F$, (6") implies

$$
\left|a_{n m}+a_{m n}\right| \leq 2\left(\left(c^{-1} \omega_{n} a_{n n}\right)\left(c \omega_{m} a_{m m}\right)\right)^{1 / 2} \leq c^{-1} \omega_{n} a_{n n}+c \omega_{m} a_{m m}
$$

for every constant $c>0, n, m \in \mathbf{N}^{*}$. Putting $c=2^{m} \omega_{2 n-2 m} \beta_{4 n-2 m}$ (resp. $c=$ $2^{m} \omega_{2 n-2 m+1} \beta_{4 n-2 m+2}$ ), (8) implies

$$
\begin{aligned}
& \beta_{4 n-2 m}\left|a_{2(n-m), 2 n}+a_{2 n, 2(n-m)}\right| \\
& \leq 2^{-m} a_{2(n-m), 2(n-m)}+2^{m} \omega_{2 n-2 m} \omega_{2 n}\left(\beta_{4 n-2 m}\right)^{2} a_{2 n, 2 n}
\end{aligned}
$$

(resp.

$$
\begin{aligned}
\beta_{4 n-2 m+2} & \left|a_{2(n-m)+1,2 n+1}+a_{2 n+1,2(n-m)+1}\right| \\
\leq & \left.2^{-m} a_{2(n-m)+1,2(n-m)+1}+2^{m} \omega_{2(n-m)+1} \omega_{2 n+1}\left(\beta_{4 n-2 m+2}\right)^{2} a_{2 n+1,2 n+1}\right),
\end{aligned}
$$

$m=1,2, \ldots, n$. Then,

$$
\begin{aligned}
S\left(f^{\sharp} f\right)= & \sum_{r=0}^{\infty} \beta_{r} T_{r}\left(\sum_{m+l=r} f_{m}^{\#} \otimes f_{l}\right)=\sum_{n=0}^{\infty} \beta_{2 n}\left(\sum_{m+l=2 n} a_{m l}\right) \\
\geqslant & \sum_{r=0}^{\infty} \beta_{2 r} a_{r r}-\sum_{n=1}^{\infty} \beta_{2 n}\left(\sum_{m=1}^{n}\left|a_{n+m, n-m}+a_{n-m, n+m}\right|\right) \\
= & \sum_{r=0}^{\infty} \beta_{2 r} a_{r r}-\sum_{n=1}^{\infty} \sum_{m=1}^{n}\left(\beta_{4 n-2 n}\left|a_{2(n-m), 2 n}+a_{2 n, 2(n-m)}\right|\right. \\
& \left.+\beta_{4 n-2 m+2}\left|a_{2(n-m)+1,2 n+1}+a_{2 n+1,2(n-m)+1}\right|\right)
\end{aligned}
$$




$$
\begin{aligned}
\geqslant & \sum_{r=0}^{\infty} \beta_{2 r} a_{r r}-\sum_{n=1}^{\infty} \sum_{m=1}^{n}\left(2^{-m} a_{2(n-m), 2(n-m)}+2^{m} \omega_{2 n-2 m} \omega_{2 n}\left(\beta_{4 n-2 m}\right)^{2} a_{2 n, 2 n}\right. \\
& \left.+2^{-m} a_{2(n-m)+1,2(n-m)+1}+2^{m} \omega_{2(n-m)+1} \omega_{2 n+1}\left(\beta_{4 n-2 m+2}\right)^{2} a_{2 n+1,2 n+1}\right) \\
\geqslant & \sum_{r=0}^{\infty}\left(\beta_{4 r} a_{2 r, 2 r}-\sum_{s=1}^{\infty} 2^{-s} a_{2 r, 2 r}-\sum_{m=1}^{r} 2^{m} \omega_{2(r-m)} \omega_{2 r}\left(\beta_{4 r-2 m}\right)^{2} a_{2 r, 2 r}\right. \\
& +\beta_{4 r+2} a_{2 r+1,2 r+1}-\sum_{s=1}^{\infty} 2^{-s} a_{2 r+1,2 r+1} \\
& \left.-\sum_{m=1}^{\mathrm{r}} 2^{m} \omega_{2(r-m)+1} \omega_{2 r+1}\left(\beta_{4 r-2 m+2}\right)^{2} a_{2 r+1,2 r+1}\right) \\
\stackrel{(7)}{=} & \sum_{r=0}^{\infty}\left(\left(\beta_{4 r}-1-\left(\beta_{4 r}-1\right)\right) a_{2 r, 2 r}+\left(\beta_{4 r+2}-1-\left(\beta_{4 r+2}-1\right)\right) a_{2 r+1,2 r+1}=0,\right.
\end{aligned}
$$

where $\sum_{m=1}^{0} .=0$, and the sums $\sum^{\infty}$. actually are finite ones due to $\operatorname{Grad}(f)<\infty$. The proof is completed.

In order to construct positive linear functionals explicitely, the following is more suitable. Let $B_{1}=\left\{b^{(\delta)} ; \delta \in \Delta\right\}$ be an (algebraic) basis of $E$, where $\Delta$ is a set of indices. Then,

$$
\boldsymbol{B}_{n}=\left\{\boldsymbol{b}^{\left(\delta_{1}\right)} \otimes \cdots \otimes \boldsymbol{b}^{\left(\delta_{n}\right)} ; \delta_{j} \in \Delta(j=1,2, \ldots, n)\right\}
$$

is a basis of $E_{n}, n \in \mathbf{N}$. Setting

$$
b^{\left(\delta_{1}, \ldots, \delta_{n}\right)}:=b^{\left(\delta_{1}\right)} \otimes \cdots \otimes b^{\left(\delta_{n}\right)}
$$

assume now that there is a sequence $\left(\omega_{n}\right)_{n=0}^{\infty}, \omega_{n}>0$, such that

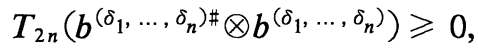

$$
\begin{aligned}
& \left|T_{n+m}\left(b^{\left(\delta_{1}, \ldots, \delta_{n}\right) \#} \otimes b^{\left(\delta_{n+1}, \ldots, \delta_{n+m}\right)}\right)\right|^{2} \leqslant \omega_{n} \omega_{m} T_{2 n}\left(b^{\left(\delta_{1}, \ldots, \delta_{n}\right) \#} \otimes b^{\left(\delta_{1}, \ldots, \delta_{n}\right)}\right) \\
& T_{2 m}\left(b^{\left(\delta_{n+1}, \ldots, \delta_{n+m}\right) \#} \otimes b^{\left(\delta_{n+1}, \ldots, \delta_{n+m}\right)}\right) \\
& \delta_{j} \in \Delta, n, m \in \mathbf{N} \text {. }
\end{aligned}
$$

Lemma 2. 5. If a linear functional $T$ satisfies $\left(6^{\prime \prime \prime}\right)$, then $T^{(\beta)}$ is $\left\{E_{\otimes}, \#\right\}$-positive, where $\left(\beta_{n}\right)_{n=0}^{\infty}$ is taken from $(7)$.

Proof. Let us be given $f=\left(f_{0}, f_{1}, \ldots\right) \in E_{\otimes}$, 


$$
f_{l}=\sum_{j=1}^{\infty} \varphi_{j}^{(l)} b_{l}^{(j)}, \varphi_{j}^{(l)} \in \mathbf{C}, b_{l}^{(j)} \in \boldsymbol{B}_{l},(l \in \mathbf{N})
$$

Then,

$$
\left.T^{(\beta)}\left(f^{\sharp} f\right)=\sum_{j=1}^{\infty} \sum_{i=1}^{\infty} \sum_{r=0}^{\infty} \sum_{m+l=r} \beta_{r} T_{r}\left(\left(\varphi_{j}^{(m)} b_{m}^{(j)}\right)\right)^{\#} \otimes \varphi_{t}^{(l)} b_{l}^{(t)}\right) .
$$

(Notice that $\sum_{J} ., \sum_{t} ., \sum_{r}$. are finite sums!) Setting

$$
a_{m, l}^{(j, t)}=T_{m+l}\left(\left(\varphi_{j}^{(m)} b_{m}^{(j)}\right)^{\#} \otimes \varphi_{t}^{(l)} b_{l}^{(t)}\right),
$$

it follows as in the proof of Lemma 2. 4 that

$$
\sum_{r=0}^{\infty} \sum_{m+l=r} \beta_{r} a_{m, l}^{(j, t)} \geqslant 0
$$

for each pair of indices $(j, t)$. Hence, $T^{(\beta)}\left(f^{\sharp} f\right) \geqslant 0$ completing the proof.

\section{§ 3. On GNS Representations of Tensor Algebras in Krein Spaces}

The aim of this section is to recall some concepts from the theory of GNS representations in a Krein space and to discuss their applications to tensor algebras.

Let us be given a Hilbert space $\mathscr{K}$, a symmetry $J$ on $\mathscr{K}$ (i. e., $J=J^{*}=J^{-1} \in$ $\mathscr{B}(\mathscr{K})$ ), and a sesquilinear form

$$
[\xi, \zeta]_{J}=(J \xi, \zeta)
$$

$\xi$, $\in \mathscr{K}$, where $(.,$.$) denotes the scalar product of \mathscr{K}$. Then, $\mathscr{K}$ equipped with $[.,]_{J}$ is called Krein space (or $J$-space).

Let $\mathscr{A}, \mathscr{B}$ be $*$-algebras with unity $\mathbb{1}$ such that $\mathbb{1} \in \mathscr{B} \subset \mathscr{A}$, and suppose that $\mathscr{B}$ is a $*$-subalgebra of $\mathscr{A}$. A linear mapping $P$ of $\mathscr{A}$ onto $\mathscr{B}$ is called abstract conditional expectation $([26])$, if

i) $P(\mathbb{1})=\mathbb{1}$,

ii) $P(a x b)=a P(x) b$ for all $a, b \in \mathscr{B}, x \in \mathscr{A}$,

iii) $P\left(x^{*}\right)=P(x)^{*}$ for all $x \in \mathscr{A}$. 
Following [1], an Hermitean and linear functional $\phi$ on $\mathscr{A}$ is said to be a $P$-functional if it satisfies
I) $\phi(P(x))=\phi(x)$,
II) $\phi\left(\left(\alpha\left(x^{*}\right)\right) x\right) \geqslant 0$,
III) $\phi(\mathbf{1})=1$

for all $x \in \mathscr{A}$, where $\alpha(x)=2 P(x)-x$ is a linear involution on $\mathscr{A}$. Further, the cone

$$
\mathscr{C}^{(\alpha)}=\left\{\sum_{i=1}^{M}\left(\alpha\left(x^{(i) *}\right)\right) x^{(i)} ; x^{(i)} \in \mathscr{A}, M \in \mathbf{N}\right\}
$$

will be refered to as the cone of generalized $\alpha$-positivity.

For $(\mathscr{A}, \mathscr{B}, P)$ and $\phi$ as above, the GNS representation for $\phi$ consists of (possibly unbounded) operators acting on a Krein space. Let us briefly recall the main steps of this construction. For details and explicite example the reader is refered to $[1 ;$ p. 270$]$ and $[14]$, respectively.

Setting

$$
\mathscr{N}_{\Phi}=\left\{x \in \mathscr{A} ; \phi\left(y^{*} x\right)=0 \text { for all } y \in \mathscr{A}\right\}
$$

and considering the residue classes $\eta_{\phi}(x), \eta_{\phi}(y)$ in the quotient space $\mathscr{A} / \mathscr{N}_{\phi}$, define

$$
\left(\eta_{\phi}(x), \eta_{\phi}(y)\right)=\phi\left(\alpha\left(y^{*}\right) x\right)
$$

Noticing that definition $\left(11^{\prime}\right)$ is correct and using $(11, \mathrm{II})$, it follows that $\left(11^{\prime}\right)$ defines a positive definite inner product on $\mathscr{A} / \mathscr{N}_{\phi}$. Let $\mathscr{K}=\left(\mathscr{A} / \mathscr{N}_{\phi}\right)^{\sim}$ (completion with respect to $\left.\|\|=.(., .)^{1 / 2}\right)$. Define then a representation $\pi_{\phi}$ of $\mathscr{A}$ on $\mathscr{K}$ with domain

$$
\begin{gathered}
D\left(\pi_{\phi}\right)=\left\{\eta_{\phi}(x) ; x \in \mathscr{A}\right\}, \\
\pi_{\phi}(x) \eta_{\phi}(y)=\eta_{\phi}(x y), \quad x, y \in \mathscr{A},
\end{gathered}
$$

and strongly cyclic vector $\eta_{\phi}(\mathbf{1})$ for $\pi_{\phi}$. Furthermore, there is also a bounded and continuous sesquilinear form

$$
\left[\eta_{\phi}(x), \eta_{\phi}(y)\right]=\phi\left(y^{*} x\right)
$$

defined on $D\left(\pi_{\phi}\right)$ and by continuous extension also on $\mathscr{K}$. Note that $\mathscr{K},[.$, 
becomes a Krein space.

The concept of *-representation $([27])$ is generalized to the one of $J$-representation $([25])$. Along these lines it is implied that $\pi_{\phi}$ is a $J$-representation of $\mathscr{A}$.

Let us now apply the concepts given above to tensor algebras. More precicely, let us first give characterizations for a graded conditional expectation $P$ on a tensor algebra $E_{\otimes}$. Using Lemma 2. 1,

$$
P=\left(P_{0}, P_{1}, P_{2}, \ldots\right)
$$

$P_{n}: E_{n} \longrightarrow E_{n}(n=0,1,2, \ldots)$, is implied. Let us introduce the subspaces

$$
\begin{aligned}
& F_{n}=\operatorname{range}\left(P_{n}\right), \\
& G_{n}=\operatorname{range}\left(I_{n}-P_{n}\right)
\end{aligned}
$$

of $E_{n}$, where $I_{n}$ denotes the identity mapping on $E_{n}, n=0,1,2, \ldots$. For any subspace $X_{n} \subset E_{n}$ let

$$
X_{n}^{h}=\left\{x_{n} \in X_{n} ; x_{n}=x_{n}^{*}\right\}
$$

denote the Hermitean part of $X_{n}$.

Proposition 3. 1. Let $P$ be a graded and linear operator on $E_{\otimes}$. The following are satisfied for $P$.

a) $P$ satisfies $(10, i)$, if and only if $P_{0}=I_{0}$.

b) The following are equivalent :

i) $P$ satisfies $(10, \mathrm{ii})$,

ii) $P_{n}\left(z_{n}^{*}\right)=\left(P_{n}\left(z_{n}\right)\right)^{*}$ for all $z_{n} \in E_{n}, n=0,1,2, \ldots\left(z_{0}^{*}=\bar{z}_{0}\right)$,

iii) $x_{n} \in F_{n}$, if and only if $x_{n}^{*} \in F_{n}$,

$y_{n} \in G_{n}$, if and only if $y_{n}^{*} \in G_{n}, n=0,1,2, \ldots$,

iv) $F_{n}=F_{n}^{h} \oplus i F_{n}^{h}, G_{n}=G_{n}^{h} \oplus i G_{n}^{h}, n=0,1,2, \ldots$,

v) $P_{n}: E_{n}^{h} \rightarrow F_{n}^{h}, n=0,1,2, \ldots$.

c) The following are equivalent :

i) (10, iii) is satisfied,

ii) $\quad \boldsymbol{P}_{r+s+t}\left(\boldsymbol{P}_{r}\left(\boldsymbol{x}_{r}\right) \otimes y_{s} \otimes \boldsymbol{P}_{t}\left(z_{t}\right)=\boldsymbol{P}_{r}\left(\boldsymbol{x}_{r}\right) \otimes \boldsymbol{P}_{s}\left(y_{s}\right) \otimes \boldsymbol{P}_{t}\left(z_{t}\right)\right.$ for all $\boldsymbol{x}_{r} \in E_{r}$, $y_{s} \in E_{s}, z_{t} \in E_{t}$, and $r, s, t \in \mathbf{N}^{*}$,

iii) for $a_{n} \in F_{n}, b_{m} \in F_{m}$, it follows $a_{n} \otimes b_{m} \in F_{n+m}$;

and if $y_{n} \in G_{n}$ then $y_{n} \otimes x_{m} \in G_{n+m}, x_{m} \otimes y_{n} \in G_{n+m}$ for all $x_{m} \in F_{m}\left(n, m \in \mathbf{N}^{*}\right)$. 
Proof. Using Lemma 2. 1, the proof is straightforward.

In order to decide whether or not the representation obtained by the construction given above is the direct sum of two independent Hilbert space representations (and thus the indefinite metric is irrelevant), S. Ôta introduced the concept of invariant dual pairs, see [1; Definition 4], [25; Definition 4. 1].

For a given subspace $\mathscr{M} \subset D\left(\pi_{\phi}\right)$, let us define

$$
E(\mathscr{M})=\left\{x \in E_{\otimes} ; \pi_{\phi}(x) \in \mathscr{M}\right\}
$$

Recall that if $\{\mathscr{M}, \mathscr{N}\}$ is a $\pi_{\phi}$-invariant dual pair, then

i) $E_{\otimes}=E(\mathscr{M})+E(\mathscr{N})$,

ii) $E(\mathscr{M}), E(\mathscr{N})$ are left-ideals in $E_{\otimes}$,

iii) $\phi\left(x^{*} x\right)>0$ for all $x \in E(\mathscr{M})$ with $x \notin \mathscr{N}_{\Phi}$ $\phi\left(x^{*} x\right)<0$ for all $x \in E(\mathscr{N})$ with $x \notin \mathscr{N}_{\phi}$,

iv) $\phi\left(y^{*} x\right)=0$ for all $x \in E(\mathscr{M}), y \in E(\mathscr{N})$, see $[1 ;$ Lemma 6$]$.

In the case of tensor algebras there is the following criterion which is easy to handle and will be used to show the absence of $\pi_{\phi}$-invariant dual pairs in the example given in $\S 5$.

Proposition 3. 2. Let us be given $e_{1}=e_{1}^{*} \in E_{1}$ with $\phi_{2}\left(e_{1} \otimes e_{1}\right)>0$. If there is some $x_{n} \in E_{n}\left(n \in \mathbf{N}^{*}\right)$ such that

a) $\phi_{n+2}\left(f_{1} \otimes x_{n} \otimes e_{1}\right) \neq 0$

for some $f_{1}=f_{1}^{*} \in E_{1}$ with $\phi_{2}\left(f_{1} \otimes f_{1}\right)<0$, or if

b) $\phi_{2 n+2}\left(e_{1} \otimes x_{n}^{*} \otimes x_{n} \otimes e_{1}\right)<0$

then there are no $\pi_{\phi}$-invariant dual pairs.

Proof. a) Assume that $\{\mathscr{M}, \mathscr{N}\}$ represents a $\pi_{\phi}$-invariant dual pair. Because of $\phi\left(\check{e}_{1}^{*} \check{e}_{1}\right)=\phi_{2}\left(e_{1}^{*} \otimes e_{1}\right)>0$ and $(12), \check{e}_{1} \in E(\mathscr{M})$ and $\left(x_{n-2} \otimes e_{1}\right)^{\vee} \in E(\mathscr{M})$ are implied. Noticing that $\breve{f}_{1} \in E(\mathscr{N}),(13)$ is a contradiction to $(12 ;$ iv)).

b) Assuming again that $\{\mathscr{M}, \mathscr{N}\}$ is a $\pi_{\phi}$-invariant dual pair, $\check{e}_{1} \in E(\mathscr{M}),\left(x_{n} \otimes e_{1}\right)^{\vee}$ $\in E(\mathscr{M})$ follow. Now, $\left(13^{\prime}\right)$ is a contradiction to $(12$, iii).

An other method to construct a Krein space theory was introduced by $L$. Jakóbczyk ([17]). There is started with a linear involution

$$
\alpha_{1}: E \longrightarrow E
$$

$\left(\alpha_{1}^{2}=I_{1}\right)$ satisfying 


$$
\alpha_{1}\left(f_{1}^{*}\right)=\left(\alpha_{1} f_{1}\right)^{*}
$$

for all $f_{1} \in E$. Setting

$$
\alpha_{n}=\alpha_{1} \otimes \cdots \otimes \alpha_{1} \quad(n \text { factors })
$$

let us introduce

$$
\alpha(f)=\left(f_{0}, \alpha_{1}\left(f_{1}\right), \alpha_{2}\left(f_{2}\right), \ldots\right)
$$

$f \in E_{\otimes}$. Then,

$$
\mathscr{K}^{(\alpha)}=\left\{\sum_{i=1}^{M} \alpha\left(f^{(i) *}\right) f^{(i)} ; f^{(i)} \in E_{\otimes}, M \in \mathbb{N}\right\}
$$

will be called the cone of $\alpha$-positivity. It is shown in [17] that every linear functional $\phi$ which is $\mathscr{K}^{(\alpha)}$-positive and $\alpha$-invariant $(\phi \circ \alpha=\phi)$ yields a representation of $E_{\otimes}$, where the field operators act on a Krein space.

Concerning the Hermiticity of $\phi$ there is the following.

Lemma 3. 3. Every $\mathscr{K}^{(\alpha)}$-positive and $\alpha$-invariant functional $\phi$ is Hermiten.

Proof. Recall that the $\alpha$-positivity of $\phi$ implies $\phi\left(\alpha\left(x^{*}\right) y\right)=\overline{\phi\left(\alpha\left(y^{*}\right) x\right)}$ for all $x, y \in E_{\otimes}$, see $[1$; Lemma 2. 1c)]. Using now the $\alpha$-invariance of $\phi$,

$$
\phi\left(x^{*} \alpha(y)\right)=\overline{\phi\left(\alpha\left(y^{*}\right) x\right)}
$$

follows. Setting $y=\mathbb{1}$ and using $\alpha(\mathbb{1})=\alpha\left(\mathbb{1}^{*}\right)=\mathbb{1}$, the assertion to be shown is implied.

In the following it will be shown that to every $\alpha$-positive $P$-functional $\phi$ on $E_{\otimes}$ there is a whole class of generalized $\alpha$-positive but not $\alpha$-positive $P$-functionals $\phi$ yielding the same GNS-respesentation.

Let us be given two tensor algebras $E_{\otimes}, \widetilde{E}_{\otimes}$, where

$$
\begin{aligned}
& \widetilde{E}=\widetilde{E}_{1}=E_{1} \oplus F_{1}, F_{1} \neq\{0\} \quad \text { (linear space), } \\
& \widetilde{E}_{2}=E_{2} \oplus\left[\left(E_{1} \otimes F_{1}\right) \oplus\left(F_{1} \otimes E_{1}\right) \oplus\left(F_{1} \otimes F_{1}\right)\right] \\
& \widetilde{E}_{n}=E_{n} \oplus\left[\left(E_{1} \otimes F_{1} \otimes \cdots \otimes F_{1}\right) \oplus \cdots \oplus\left(F_{1} \otimes \cdots \otimes F_{1}\right)\right],
\end{aligned}
$$


$n=3,4, \ldots$, and all of the summands in $[\cdots]$ of (15) only contain factors $E_{1}, F_{1}$, but there is at least one factor $F_{1}$ in each summand. Let us abbreviate

$$
\widetilde{E}_{n}=E_{n} \oplus F_{n},
$$

where $F_{n}=[\cdots]$ is taken from (15). Setting $F=F_{1} \oplus F_{2} \oplus \cdots$,

$$
\widetilde{E}_{\otimes}=E_{\otimes} \oplus F
$$

follows. Furthermore, let $\phi$ be a $\mathscr{K}^{(\alpha)}$-positive linear functional on $E_{\otimes}$. Let us consider the liner extension $\tilde{\phi}$ of $\phi$ from $E_{\otimes}$ onto $\widetilde{E}_{\otimes}$ given by

$$
\tilde{\phi}(x)=\left\{\begin{array}{ccc}
\phi(x) & \text { for } & x \in E_{\otimes} \\
0 & \text { for } & x \in F .
\end{array}\right.
$$

This extension $\bar{\phi}$ is called the trivial one. (For a further discussion of such an extension, the reader is refered to [32].)

Proposition 3. 4. Let us be given a P-functional $\phi$ on $E_{\otimes}$ such that $\phi$ is $\mathscr{K}^{(\alpha)}$-positive. Then, every trivial extension $\tilde{\phi}$ onto $\widetilde{E}_{\otimes}$ is a P-functional not being $\mathscr{K}^{(\alpha)}$-positive.

Proof. Using $P_{n}\left(x_{n}\right)=\frac{1}{2}\left(\alpha_{n}\left(x_{n}\right)+x_{n}\right), x_{n} \in E_{n}(n=1,2, \ldots)$, let us define projections

$$
\widetilde{P}_{n}\left(x_{n}\right)=\left\{\begin{array}{cl}
P_{n}\left(x_{n}\right) & \text { for } x_{n} \in E_{n} \\
0 & \text { for } x_{n} \in F_{n},
\end{array}\right.
$$

$\widetilde{P}=\left(I_{0}, \widetilde{P}_{1}, \widetilde{P}_{2}, \ldots\right)$. Define further

$$
\tilde{\alpha}_{n}=2 \widetilde{P}_{n}-\widetilde{I}_{n}, \quad \tilde{\alpha}=\left(I_{0}, \tilde{\alpha}_{1}, \tilde{\alpha}_{2}, \ldots\right) .
$$

It is straightforward to show that $\tilde{\phi}\left(\mathscr{C}^{(\alpha)}\right) \geqslant 0$ and $\phi(\widetilde{P} f)=\phi(f), f \in \widetilde{E}_{\otimes}$. Hence, $\tilde{\phi}$ is a $P$-functional on $\widetilde{E}_{\otimes}$. Consider $0 \neq y_{1} \in F_{1}$. Then,

$$
\begin{gathered}
\left(\tilde{\alpha}_{1} \otimes \tilde{\alpha}_{1}\right)\left(y_{1} \otimes y_{1}\right)=\left(2 \widetilde{P}_{1}-I_{1}\right) y_{1} \otimes\left(2 \widetilde{P}_{1}-I_{1}\right) y_{1}=y_{1} \otimes y_{1}, \\
\tilde{\alpha}_{2}\left(y_{1} \otimes y_{1}\right)=\left(2 \widetilde{P}_{2}-I_{2}\right)\left(y_{1} \otimes y_{1}\right)=-y_{1} \otimes y_{1},
\end{gathered}
$$

imply that $\tilde{\alpha}_{2} \neq \tilde{\alpha}_{1} \otimes \tilde{\alpha}_{1}$. Hence, $\mathscr{C}^{(\tilde{\alpha})}$ is not any cone of $\alpha$-positivity. 
Corollary 3. 5. The GNS-representations obtained from $\phi$ and $\tilde{\phi}$ are unitarily equivalent.

Proof. Consider

$$
\begin{array}{ll}
\mathcal{N}_{\Phi}=\left\{x \in E_{\otimes} ; \phi\left(y^{*} x\right)=0\right. & \text { for all } \left.y \in E_{\otimes}\right\}, \\
\mathcal{N}_{\tilde{\phi}}=\left\{x \in \widetilde{E}_{\otimes} ; \tilde{\phi}\left(y^{*} x\right)=0\right. & \text { for all } \left.y \in \widetilde{E}_{\otimes}\right\} .
\end{array}
$$

Using that $F$ defined beforehand of $\left(15^{\prime \prime}\right)$ is a two-sided ideal in $E_{\otimes}$ and that $\tilde{\phi}(f)$ $=0$ for all $f \in F$, it follows that

$$
\tilde{\phi}\left(\tilde{y}^{*} \tilde{x}\right)=\phi\left(y^{*} x\right)
$$

where $\tilde{y}=y+g, \tilde{x}=x+f, y \in E_{\otimes}, x \in \mathcal{N}_{\phi}, g, f \in F . \quad$ Hence, $\mathscr{N}_{\tilde{\phi}}=\mathcal{N}_{\phi}+F . \quad$ The assertion under consideration is now obvious.

Remark 3. 6. a) Sometimes the following minor generalization of the concept of $\alpha$-positivity, which is also refered to as $\alpha$-positivity in some notes, is considered (see [18; Definition III. 3]). For distinction, let us write respectively, $\tilde{\alpha}$-positivity for this concept and cone of $\tilde{\alpha}$-positivity for the corresponding cone $\mathscr{K}^{(\tilde{\alpha})}$. Generalizing (14), $\ldots,\left(14^{\prime \prime}\right)$, here it is started with a $*$-automorphism $\tilde{\alpha}: E_{\otimes} \longrightarrow$ $E_{\otimes}$. Noting that $\tilde{\alpha}$ represents a $*$-automorphism on $E_{\otimes}$, if and only if

$$
\widetilde{P}(f g)=\widetilde{P}(f) \widetilde{P}(g)+\widetilde{Q}(f) \widetilde{Q}(g), \widetilde{P}\left(f^{*}\right)=\widetilde{P}(f)^{*}
$$

for all $f, g \in E_{\otimes}$, where $\widetilde{P}=\frac{1}{2}(\tilde{\alpha}+I), \widetilde{P}+\widetilde{Q}=I$, it follows straightforwardly that $\widetilde{P}$ is an abstract conditional expectation. Hence, every functional satisfying [18; Def. III. 2] is a $P$-functional, and thus yields a GNS representation of $E_{\otimes}$ by operators acting on a Krein space.

b) Obviously, the concept of $\tilde{\alpha}$-positivity is between the one of $\alpha$-positivity and of generalized $\alpha$-positivity, i.e. every functional being $\alpha$-positive is $\tilde{\alpha}$-positive, and further, every $\tilde{\alpha}$-positive functional is generalized $\alpha$-positive.

c) A further discussion on this concept is given in Corollary 4. 6, and it will be shown there that the concepts of $\alpha$-positivity and $\tilde{\alpha}$-positivity coincide in the most interesting cases.

\section{$\S$ 4. On Cones of $\alpha$-Positivity and Generalized $\alpha$-Positivity in Tensor Algebras}

For the following let us be given any tensor algebra $E_{\otimes}$. If a linear involution $\alpha$ is given on $E_{\otimes}$, then let us define an antilinear bijection "\#” by 


$$
f^{\#}=(\alpha(f))^{*},
$$

$f \in E_{\otimes}$. The restriction of \# to subspaces $E_{n}\left(n \in \mathbf{N}^{*}\right)$ will also be denoted by \#.

The following proposition and example are concerned with relations between $\alpha$-positive (resp. generalized $\alpha$-positive) cones, alg-\# cones and involutive cones.

Proposition 4. 1. a) Every cone $\mathscr{K}^{(\alpha)}$ of $\alpha$-positivity is an involutive cone with $\mathscr{K}^{(\alpha)}=\left\{\boldsymbol{E}_{\otimes}, \#\right\}$.

b) Let us be given a cone of generalized $\alpha$-positivity $\mathscr{C}^{(\alpha)}$.

The following are equivalent.

i) $\mathscr{C}^{(\alpha)}$ is an alg-\# cone, (where \# is given in (16)),

ii) $P Q_{n}=Q_{n} P, n=0,1,2, \ldots$, where $P=\frac{1}{2}(\alpha+I)$,

iii) the projection $P$ is graded,

iv) the involution $\alpha$ is graded.

Proof. a) Let us be given $\mathscr{K}^{(\alpha)}$. Using (14), (14'), (14") and (10),

$$
\left(f^{\#}\right)_{n}=\left(\alpha_{n}\left(f_{n}\right)\right)^{*}=\left(f_{n}\right)^{\#}, n=0,1,2, \ldots,
$$

follow for all $f \in E_{\otimes}$. Hence, the mapping \# is graded. Using Lemma 2. 1, (4') follows. Noticing $\left(\alpha_{n}\left(f_{n}\right)\right)^{*}=\alpha_{n}\left(f_{n}^{*}\right)$ and using $\alpha^{2}=I$, (4) is implied. Using now the antilinearity of \#, it is sufficient to prove (5) for monomials $f_{n} \otimes g_{m}, f_{n} \in E_{n}, g_{m}$ $\in \boldsymbol{E}_{m}\left(m, n \in \mathscr{N}^{*}\right)$. Consequently,

$$
\left(f_{n} \otimes g_{m}\right)^{\#}=\alpha_{n+m}\left(g_{m}^{*} \otimes f_{n}^{*}\right)=\alpha_{m}\left(g_{m}^{*}\right) \otimes \alpha_{n}\left(f_{n}^{*}\right)=g_{m}^{\#} \otimes f_{n}^{\#}
$$

yield (5). Hence $\mathscr{K}^{(\alpha)}=\left\{\boldsymbol{E}_{\otimes}, \#\right\}$ represents an involutive cone.

b) i) $\Rightarrow$ ii): Assuming i), inserting $\alpha=2 P-I$ in $\left(4^{\prime}\right)$, and using $Q_{n}\left(f^{*}\right)=$ $\left(Q_{n} f\right)^{*}$, ii) follows straightforwardly.

ii) $\Rightarrow$ i): Noticing that (10iii) implies $\alpha\left(f^{*}\right)=(\alpha(f))^{*}$,

$$
f^{\# \#}=\left(\alpha\left(\alpha(f)^{*}\right)\right)^{*}=\alpha^{2}(f)=f,
$$

$f \in E_{\otimes}$, follow. Assuming now ii), it follows that

$$
\begin{aligned}
\left(Q_{n} f\right)^{\#} & =\left(\alpha\left(Q_{n} f\right)\right)^{*}=\left((2 P-I) Q_{n} f\right)^{*}=\left(Q_{n}(2 P-I) f\right)^{*} \\
& =\left(Q_{n} \alpha(f)\right)^{*}=Q_{n}\left((\alpha(f))^{*}\right)=Q_{n}\left(f^{\sharp}\right),
\end{aligned}
$$

$f \in E_{\otimes}, n \in \mathbf{N}^{*}$. Hence $\mathscr{C}^{(\alpha)}=\left\{\boldsymbol{E}_{\otimes}, \#\right\}$ is an alg-\# cone. The equivalences of ii) $\Leftrightarrow$ iii) and ii) $\Leftrightarrow$ iv) are immediate consequences of Lemma 2. 1 . 
The following example shows that there are also cones of generalized $\alpha$-positivity not being alg-\# cones.

Example 4. 2. Consider the tensor algebra

$$
\mathbf{C}_{\otimes}=\mathbf{C}_{0} \oplus \mathbf{C}_{1} \oplus \mathbf{C}_{2} \oplus \cdots,
$$

where $\mathbb{C}_{j}=\mathbb{C}(j=0,1,2, \ldots)$. Define an involution on $\mathbb{C}_{\otimes}$ by setting

$$
\alpha(x)=\left(2\left(\sum_{i=0}^{\infty} x_{i}\right)-x_{0},-x_{1},-x_{2}, \ldots\right),
$$

and notice that $\alpha$ defines a projection $P: \mathbb{C}_{\otimes} \longrightarrow \check{\mathbf{C}}_{0}$, where

$$
P(x)=\left(\sum_{i=0}^{\infty} x_{i}, 0,0, \ldots\right)
$$

$x \in \mathbf{C}_{\otimes}$. It is straightforward to show that $P$ is a conditional expectation, where $\mathscr{A}$ $=\mathbb{C}_{\otimes}$ and $\mathscr{B}=\check{\mathbf{C}}_{0}$ in the setting beforehand of (10). Hence,

$$
\mathscr{C}^{(\alpha)}=\left\{\sum_{i=1}^{M}\left(\alpha\left(f^{(i)}\right)^{*} f^{(i)} ; f^{(i)} \in \mathbf{C}_{\otimes}, M \in \mathbb{N}\right\}\right.
$$

represents a cone of generalized $\alpha$-positivity. However noticing that

$$
P Q_{n} \neq Q_{n} P
$$

$n \in \mathbb{N}^{*}, \mathscr{C}^{(\alpha)}$ is no alg-\# cone due to Proposition 4. 1b).

Let us now investigate the GNS-representation $\pi_{\phi}$ of $\mathbb{C}_{\otimes}$ for any $P$-functional $\phi$, where $\alpha$ and $P$ are given above. Recall that there is a $*$-isomorphism between $\mathbf{C}_{\otimes}$ and $\mathbf{C}[t]$ (algebra of polynomials in one (real) variable $t$ given by

$$
\mathbb{C}_{\otimes} \ni x \longleftrightarrow \hat{x}(t) \in \mathbf{C}[t],
$$

where $\hat{x}(t)=\sum_{n=0}^{\infty} x_{n} t^{n},(x+y)^{\wedge}(t)=\hat{x}(t)+\hat{y}(t),(x y)^{\wedge}(t)=\hat{x}(t) \hat{y}(t),\left(x^{*}\right)^{\wedge}(t)=$ $\overline{\hat{x}(t)}, x=\left(x_{0}, x_{1}, \ldots\right), y \in \mathbb{C}_{\otimes}$. Note that

$$
(\boldsymbol{P}(\boldsymbol{x}))^{\wedge}=\hat{\boldsymbol{x}}(1)
$$

Using (11I, III), it follows that

$$
\phi(x)=\sum_{i=0}^{\infty} \phi_{i}\left(x_{i}\right)=\phi_{0}\left(\sum_{i=0}^{\infty} x_{i}\right)=\sum_{i=0}^{\infty} x_{i}=\hat{x}(1),
$$

$x \in \mathbf{C}_{\otimes}$. The left kernel $\mathscr{N}_{\phi}$ of $\phi$ is then given by 


$$
\mathcal{N}_{\Phi}=\left\{\boldsymbol{x} \in \mathbf{C}_{\otimes} ; \phi\left(y^{*} \boldsymbol{x}\right)=0 \text { for all } y \in \mathbf{C}_{\otimes}\right\}=\left\{\boldsymbol{x} \in \mathbf{C}_{\otimes} ; \hat{x}(1)=0\right\}
$$

Thus, $D\left(\pi_{\phi}\right)=\mathbf{C}_{\otimes} / \mathscr{N}_{\phi} \cong \mathbf{C}$ and $\operatorname{dim}\left(D\left(\pi_{\phi}\right)\right)=1$. Hence there is no chance to get an indefinite metric on $D\left(\pi_{\phi}\right)$. Furthermore,

$$
\pi_{\phi}(x) \eta_{\phi}(y)=\eta_{\phi}(x y) \longleftrightarrow \hat{x}(1) \hat{y}(1),
$$

$x \in \mathbf{C}_{\otimes}, \eta_{\phi}(\cdot) \in D\left(\pi_{\phi}\right)$. It holds further

$$
\begin{aligned}
& {[x, x]=\phi\left(x^{*} x\right)=|\hat{x}(1)|^{2} .} \\
& (x, x)=\phi\left(x^{\#} x\right)=\phi\left((\alpha x)^{*} x\right)=\overline{(2 \hat{x}(1)-x(t))} \hat{x}(t)_{\mid t=1}=|\hat{x}(1)|^{2} .
\end{aligned}
$$

Thut, $[x, x]=(x, x) \geqslant 0$ showing also that there is no indefinite metric in the theory obtained in that way.

The following is concerned with relations between the cones of $\alpha$-positivity and those of generalized $\alpha$-positivity. Let us start with some definitions and general considerations needed in the proof of Proposition 4. 5.

For a linear involution $\alpha$ given on $E_{\otimes}$ let us consider the fixed point set of $\alpha$

$$
\mathscr{F}=\left\{f \in E_{\otimes} ; \alpha(f)=f\right\},
$$

and the following subspaces

$$
\begin{aligned}
\mathscr{F}_{1}= & \left\{f_{1} \in E_{1} ; \alpha\left(f_{1}\right)=f_{1}\right\} \\
\mathscr{G}_{1}= & \left\{f_{1} \in E_{1} ; \alpha\left(f_{1}\right)=-f_{1}\right\}, \\
\mathscr{F}_{n}= & \operatorname{span}\left\{e^{(1)} \otimes \cdots \otimes e^{(n)} ; e^{(j)} \in \mathscr{F}_{1} \cup \mathscr{G}_{1}, \text { the number of } e^{(j)}\right. \text { with } \\
& \left.e^{(j)} \in \mathscr{G}_{1} \text { is } 0 \text { or even }(j=1,2, \ldots n)\right\} \\
\mathscr{G}_{n}= & \operatorname{span}\left\{e^{(1)} \otimes \cdots \otimes e^{(n)} ; e^{(j)} \in \mathscr{F}_{1} \cup \mathscr{G}_{1}, \text { the number of } e^{(j)}\right. \text { with } \\
& \left.e^{(j)} \in \mathscr{G}_{1} \text { is odd }(j=1,2, \ldots, n)\right\}
\end{aligned}
$$

$n=2,3, \ldots$. Set further $\mathscr{F}_{0}=\mathbf{C}, \mathscr{G}_{0}=\{0\}$. It is straightforward to show the following.

Lemma 4. 3. If $\alpha$ is graded, then the (algebraic) direct decompositions $E_{m}=\mathscr{G}_{m}$ $\oplus \mathscr{F}_{m}, m=0,1,2, \ldots$, follow. 
Remark. i) Considering Example 4.2, it follows that the involution $\alpha$ constructed there is not graded. Further, $\mathscr{F}_{1}=\mathscr{G}_{1}=\{0\}$. Hence, the assertion of Lemma 4. 3 is not satisfied. The assumption of the gradedness of $\alpha$ is thus not redundant. ii) If $\alpha$ is graded and $\alpha=\left(I_{0}, \alpha_{1}, \alpha_{2}, \ldots\right)$, then $\mathscr{F}_{n}$ is the fixed point set of $\alpha_{1}^{\otimes n}=\alpha_{1} \otimes \cdots \otimes \alpha_{1}$ ( $n$ factors). Furthermore,

$$
\mathscr{G}_{n}=\left\{f_{n} \in E_{n} ; \alpha_{1}^{\otimes n} f_{n}=-f_{n}\right\}, n=1,2, \ldots
$$

For all that follows now assume that $\alpha$ is graded. Let us relate projections to the subspaces given above. Consider operators $P_{n, \mu}: E_{n} \longrightarrow E_{n}$ defined by

$$
\begin{aligned}
P_{n, \mu}= & P_{1} \otimes \cdots \otimes P_{1} \otimes I_{1} \otimes \cdots \otimes I_{1}+P_{1} \otimes \cdots \otimes I_{1} \otimes P_{1} \otimes \cdots \otimes I_{1} \\
& +\cdots+I_{1} \otimes \cdots \otimes I_{1} \otimes P_{1} \otimes \cdots \otimes P_{1},
\end{aligned}
$$

where the sum on the right-hand side of (17) is over all the $\left(\begin{array}{l}n \\ \mu\end{array}\right)$ summands of $\mu$ factors $I_{1}$ and $n-\mu$ factors $P_{1}=\frac{1}{2}\left(\alpha_{1}+I_{1}\right), \mu=0,1,2, \ldots, n$. Set further

$$
\boldsymbol{P}_{n}=\frac{1}{2}\left(\sum_{\mu=0}^{n} 2^{n-\mu}(-1)^{\mu} \boldsymbol{P}_{n, \mu}+\boldsymbol{I}_{n}\right),
$$

$n=0,1,2, \ldots$

Lemma 4. 4. The operators $P_{n}: E_{n} \longrightarrow E_{n}$ are the projections onto $\mathscr{F}_{n}$ with respect to the decomposition $E_{n}=\mathscr{F}_{n} \oplus \mathscr{G}_{n}, n=1,2, \ldots$.

Proof. Using $\alpha_{1}=2 P_{1}-I_{1}$, it follows that

$$
\alpha_{1}^{\otimes n}=\left(2 P_{1}-I_{1}\right) \otimes \cdots \otimes\left(2 P_{1}-I_{1}\right)=\sum_{\mu=0}^{n} 2^{n-\mu}(-1)^{\mu} P_{n, \mu}=2 P_{n}-I_{n} .
$$

Recalling ii) of the remark to Lemma 4. 3, (18) implies the assertion to be shown.

Proposition 4. 5. a) Every cone of $\alpha$-positivity is also a cone of generalized $\alpha$-positivity.

b) Let us be given a cone $\mathscr{C}^{(\alpha)}$ of generalized $\alpha$-positivity.

The following are then equivalent.

i) $\mathscr{C}^{(\alpha)}$ is a cone of $\alpha$-positivity,

ii) $\mathscr{C}^{(\alpha)}$ is an involutive cone,

iii) the involution $\alpha$ is graded and there is a projector $P_{1}: E_{1} \longrightarrow E_{1}$ such that $\alpha_{n}=2 P_{n}-I_{n}$, where $P_{n}$ is given in $\left(17^{\prime}\right), n=1,2 \ldots$,

iv) the fixed point set of $\alpha$ satisfies $\mathscr{F}=\bigoplus_{n=0}^{\infty} \mathscr{F}_{n}$, where $\mathscr{F}_{n}$, $\mathscr{F}$ are given beforehand of Lemma 4. 3. 
Proof. a) Let us be given a cone $\mathscr{K}^{(\alpha)}$ of $\alpha$-positivity. Then, $\alpha=\left(\alpha_{0}, \alpha_{1}, \alpha_{2}\right.$, $\ldots)$ with $\alpha_{n}=\alpha_{1}^{\otimes n}(n=1,2, \ldots), \alpha_{0}=I_{0}$. Recalling that a projector $P_{n}=\frac{1}{2}\left(\alpha_{n}+I_{n}\right)$ belongs to every involution $\alpha_{n},\left(10, \mathrm{i}\right.$, iii) are satisfied for $P=\left(I_{0}, P_{1}, P_{2}, \ldots\right)$ because of Proposition 3.1a) and (14), (14'). Using the linearity of $P$, it is sufficient to show $\left(10\right.$, ii) for monomials $a_{n} \otimes x_{l} \otimes b_{m}$, where $a_{n} \in \operatorname{range}\left(P_{n}\right), b_{m} \in$ $\operatorname{range}\left(P_{m}\right), x_{l} \in E_{l}, m, n, l \in \mathbf{N}^{*}$. Noticing that $\alpha_{n}\left(a_{n}\right)=a_{n}$ and $\alpha_{m}\left(b_{m}\right)=b_{m}, \quad(10$, ii) follows from

$$
\begin{aligned}
P_{n+l+m}\left(a_{n} \otimes x_{l} \otimes b_{m}\right) & =\frac{1}{2}\left(\alpha_{n}\left(a_{n}\right) \otimes \alpha_{l}\left(x_{l}\right) \otimes \alpha_{m}\left(b_{m}\right)+a_{n} \otimes x_{l} \otimes b_{m}\right) \\
& =\frac{1}{2}\left(a_{n} \otimes\left(\alpha_{l}\left(x_{l}\right)+x_{l}\right) \otimes b_{m}\right)=a_{n} \otimes P_{l}\left(x_{l}\right) \otimes b_{m} .
\end{aligned}
$$

b) i) $\Rightarrow$ iv) $\Rightarrow$ iii) are immediate consequences of the remark ii) to Lemma 4.3 and of Lemma 4. 4. iii) $\Rightarrow$ ii) : Using (18),

$$
\alpha_{n}=2 P_{n}-I_{n}=\alpha_{1}^{\otimes n}
$$

$n=1,2, \ldots$, are implied. Analogously to the proof of Proposition 4. 1b), it is now shown that $\mathscr{C}^{(\alpha)}$ is an involutive cone. ii) $\Rightarrow$ i) : Assuming ii), Proposition 4. 1b) implies that $\alpha=\left(I_{0}, \alpha_{1}, \alpha_{2}, \ldots\right)$ is graded. The further proof is given by induction. Assume that $\alpha_{n}=\alpha_{1}^{\otimes n}$ for some $n \in \mathbf{N}$. Recalling that (10, iii) implies $\alpha_{n+1}\left(f_{n+1}^{*}\right)=$ $\alpha_{n+1}\left(f_{n+1}\right)^{*}, f_{n+1} \in E_{n+1}$, it follows that

$$
\begin{aligned}
\alpha_{n+1}\left(g_{1} \otimes f_{n}\right) & =\alpha_{n+1}\left(f_{n}^{*} \otimes g_{1}^{*}\right) \stackrel{(16)}{=}\left(f_{n} * \otimes g_{1}^{*}\right) \stackrel{(5)}{=} \\
& =\left(g_{1}^{*}\right)^{\#} \otimes\left(f_{n}^{*}\right)^{\#}=\alpha_{1}\left(g_{1}\right) \otimes \alpha_{n}\left(f_{n}\right)
\end{aligned}
$$

for all $g_{1} \in E_{1}, f_{n} \in E_{n}$. Hence, $\alpha_{n+1}=\alpha_{1}^{\otimes(n+1)}$. Consequently, $\mathscr{C}^{(\alpha)}$ is a cone of $\alpha$-positivity.

\section{Recalling Remark 3.6, the following holds.}

Corollary 4. 6. Let us be given a cone $\mathscr{K}^{(\tilde{\alpha})}$ of $\tilde{\alpha}$-positivity. Then, $\mathscr{K}^{(\tilde{\alpha})}$ is a cone of $\alpha$-positivity, if and only if $\mathscr{K}^{(\tilde{\alpha})}$ is an alg-\# cone.

Proof. $(\Rightarrow)$ : Assuming that $\mathscr{K}^{(\tilde{\alpha})}$ is a cone of $\alpha$-positivity, Proposition 4. 1a) implies that $\mathscr{K}^{(\tilde{\alpha})}$ is an involutive cone, and thus an alg-\# cone. $(\Leftarrow)$ : Assume now that $\mathscr{K}^{(\tilde{\alpha})}$ is an alg-\# cone. Remark 3.6b) and Proposition 4. 1b) imply that $\tilde{\alpha}$ is graded. Using that $\tilde{\alpha}$ is a $*$-automorphism, it follows that $\mathscr{K}^{(\tilde{\alpha})}$ is an involutive cone, and hence a cone of $\alpha$-positivity due to Proposition 4. 5b). 
Remark. Noticing that the cone $\mathscr{C}^{(\alpha)}$ constructed in Example 4. 2 is a cone of $\tilde{\alpha}$-positivity too, it follows that there are cones of $\tilde{\alpha}$-positivity not being alg-\# cones.

The following example shows that there are cones $\mathscr{C}^{(\alpha)}$ of generalized $\alpha$-positivity which are alg-\# cones but not involutive ones. Applying Proposition 4. 5b), it follows that these cones are not any cones of $\alpha$-positivity.

Example 4. 7. Let us be given a tensor algebra $E_{\otimes}$ with $\operatorname{dim}(E) \geqslant 2$, and a projection

$$
P=\left(I_{0}, P_{1}, P_{2}, \ldots\right)
$$

on $E_{\otimes}$, where $P_{1}\left(x_{1}^{*}\right)=P\left(x_{1}\right)^{*}, x_{1} \in E, 0 \neq P_{1} \neq I_{1}$, and

$$
P_{n}=P_{1} \otimes \ldots \otimes P_{1} \quad(n \text { factors }),
$$

$n=1,2, \ldots$ Obviously, $(10, \mathrm{i}, \mathrm{ii}, \mathrm{iii})$ and $P Q_{n}=Q_{n} P \quad\left(n \in \mathbb{N}^{*}\right)$ are satisfied. Applying now Proposition 4. 1b), $\mathscr{C}^{(\alpha)}=\left\{E_{\otimes}, \#\right\}$ is an alg-\# cone, where $f^{\#}=$ $\alpha\left(f^{*}\right), \alpha=2 P-I, f \in E_{\otimes}$.

Considering $\alpha_{2}=2 P_{1} \otimes P_{1}-I_{2}$, it follows that

$$
\left(f_{1} \otimes g_{1}\right)^{\#}=\alpha_{2}\left(f_{1} \otimes g_{1}\right)^{*}=\alpha_{2}\left(g_{1}^{*} \otimes f_{1}^{*}\right)=2 P_{1}\left(g_{1}^{*}\right) \otimes P_{1}\left(f_{1}\right)^{*}-g_{1}^{*} \otimes f_{1}^{*},
$$

$f_{1}, g_{1} \in E_{1}$. Assuming now that $\mathscr{C}^{(\alpha)}$ were an involutive cone,

$$
\left(f_{1} \otimes g_{1}\right)^{\#}=g_{1}^{\#} \otimes f_{1}^{\#}=\left(2 P_{1}\left(g_{1}^{*}\right)-g_{1}^{*}\right) \otimes\left(2 P_{1}\left(f_{1}^{*}\right)-f_{1}^{*}\right)
$$

would be implied. If $f_{1}, g_{1} \in E_{1}$ with $g_{1} \neq 0, P_{1}\left(g_{1}\right)=0,0 \neq f_{1} \neq P_{1}\left(f_{1}\right)$ are inserted in $(19)$ and $\left(19^{\prime}\right)$; then the contradiction

$$
g_{1}^{*} \otimes\left(f_{1}-P_{1}\left(f_{1}\right)\right) *=0
$$

follows. Hence, $\mathscr{C}^{(\alpha)}$ is not any involutive cone.

The following lemma shows however that the projection $P$ introduced above cannot be used to construct a nontrivial Krein-space theory by the GNS representation recalled in $\S 3$.

Lemma. Let $\phi$ be some $P$-functional, where $P$ is taken from Example 4. 7. Constructing the GNS representation, the inner product $\left[\eta_{\phi}(x), \eta_{\phi}(y)\right]=\phi\left(y^{*} x\right)$, $x, y \in E_{\otimes}$, is positive definite (i.e., the Krein space $\mathscr{H}=D\left(\pi_{\phi}\right)^{\sim}$ is a Hilbert space).

Proof. Noticing that $P\left(f^{*} g\right)=P\left(f^{*}\right) P(g), f, g \in E_{\otimes}$, it follows from (11, I, 
II) that

$$
\begin{aligned}
{\left[\eta_{\phi}(x), \eta_{\phi}(x)\right] } & =\phi\left(x^{*} x\right)=\phi\left(P\left(x^{*} x\right)\right)=\phi\left(P(x)^{*} P(x)\right) \\
& =\phi\left((\alpha(P(x)))^{*} P(x)\right)=\phi\left((P(x))^{\#} P(x)\right) \geqslant 0
\end{aligned}
$$

for all $x \in E_{\otimes}$. This completes the proof.

\section{$\S$ 5. Construction of a $P$-Functional not Being $\alpha$-Positive}

Examples 4.2 and 4.7 provide $P$-functionals which are not $\alpha$-positive. However, it is explained there that these examples cannot be used to construct nontrivial Krein-space theories by GNS representation. The aim of this $\S$ is to construct an example of a $P$-functional which is not $\alpha$-positive and gives a $\mathrm{Krein}$ space theory with indefinite metric via the GNS representation recalled in $\S 3$. This is an answer to question $(Q)$ formulated in $\S 1$.

Let us consider the tensor algebra $\left(\mathbf{C}^{2}\right)_{\otimes}$ over the basic space $\mathbf{C}^{2}$. Further, let $e^{(i)}=e^{(i)} *(i=1,2)$ be a basis of $\mathbf{C}^{2}$. Recall that a basis of $E_{m}$ is given by

$$
\boldsymbol{B}_{m}=\left\{e^{\left(i_{1}\right)} \otimes \cdots \otimes e^{\left(i_{m}\right)} ; i_{j} \in\{1,2\} \quad(j=1,2, \ldots, m)\right\},
$$

$m=1,2, \ldots$.

In order to define an abstract conditional expectation on $\left(\mathbf{C}^{2}\right)_{\otimes}$, let us introduce sets $X_{n} \subset E_{n}\left(n \in \mathbf{N}^{*}\right)$ by setting

$$
\begin{aligned}
X_{0}= & \{1\}, \quad X_{1}=\left\{e^{(1)}\right\}, \\
X_{n}= & \left\{e^{\left(i_{1}\right)} \otimes \cdots \otimes e^{\left(i_{n}\right)} ; i_{j} \in\{1,2\} \quad(j=1,2, \ldots, n), e^{(2)}\right. \text { occurs only in } \\
& \text { pairs of two neighboring elements } \left.e^{(2)}\right\} .
\end{aligned}
$$

(Notice that, e.g., $X_{2}=\left\{e^{(1)} \otimes e^{(1)}, e^{(2)} \otimes e^{(2)}\right\}$,

$$
\left.X_{3}=\left\{e^{(1)} \otimes e^{(1)} \otimes e^{(1)}, e^{(1)} \otimes e^{(2)} \otimes e^{(2)}, e^{(2)} \otimes e^{(2)} \otimes e^{(1)}\right\} .\right)
$$

The sets $X_{n}$ satisfy the following properties that are the key for the construction of the $P$-functional given in this $\S$.

Lemma 5. 1. a) If $x_{m} \in X_{m}, \tilde{x}_{n} \in X_{n}$, then $x_{m} \otimes \tilde{x}_{n} \in X_{n+m}$; and if $y_{n} \in B_{n} \backslash X_{n}$, then $y_{n} \otimes z_{m} \in B_{n+m} \backslash X_{n+m}, z_{m} \otimes y_{n} \in B_{n+m} \backslash X_{n+m}$ for all $z_{m} \in X_{m}\left(n, m \in \mathbf{N}^{*}\right)$. $X_{2 n}$.

b) If $a_{m}^{*} \otimes \tilde{a}_{n} \in X_{m+n}$ for $a_{m} \in B_{m}, \tilde{a}_{n} \in B_{n}$, then $a_{m}^{*} \otimes a_{m} \in X_{2 m}$ and $a_{n}^{*} \otimes a_{n} \in$ 
The proof follows straightforwardly.

Let us define

$$
P_{m}\left(b_{m}\right)=\left\{\begin{array}{lll}
b_{m} & \text { if } & b_{m} \in X_{m} \\
0 & \text { if } & b_{m} \in B_{m} \backslash X_{m}
\end{array}\right.
$$

$m=1,2, \ldots$

Proposition 5. 2. $P=\left(I_{0}, P_{1}, P_{2}, \ldots\right)$ is an abstract conditional expectation.

Proof. (10, i) follows from $P_{0}=I_{0}$ and Proposition 3. 1a). Noticing

$$
X_{n}=X_{n}^{*}\left(=:\left\{x_{n}^{*} ; x_{n} \in X_{n}\right\}\right),
$$

$n \in \mathbf{N}^{*}$, (21) implies (10, iii). Using Lemma 5. 1a) and range $\left(P_{l}\right)=X_{l}\left(l \in \mathbf{N}^{*}\right)$, Proposition 3. 1c) yields (10, ii).

Lemma 5. 3. $\mathscr{C}^{(\alpha)}=\left\{\sum_{i=1}^{M} \alpha\left(x^{(l)}\right)^{*} x^{(i)} ; x^{(l)} \in\left(\mathbb{C}^{2}\right)_{\otimes}, M \in \mathbf{N}\right\}$ is not any cone of $\alpha$ positivity, where $\alpha=2 P-I$.

Proof. Notice that

$$
\alpha_{n}\left(b_{n}\right)=\left\{\begin{array}{rll}
b_{n} & \text { if } & b_{n} \in X_{n} \\
-b_{n} & \text { if } & b_{n} \in B_{n} \backslash X_{n}
\end{array}\right.
$$

follows from (21). Then,

$$
\begin{aligned}
& \alpha_{3}\left(e^{(2)} \otimes e^{(1)} \otimes e^{(2)}\right)^{*}=-e^{(2)} \otimes e^{(1)} \otimes e^{(2)}, \\
& \alpha_{1}\left(e^{(2)}\right)^{*}=-e^{(2)}, \quad \alpha_{2}\left(e^{(1)} \otimes e^{(2)}\right)^{*}=-e^{(2)} \otimes e^{(1)}
\end{aligned}
$$

are implied. Hence,

$$
\alpha_{3}\left(e^{(2)} \otimes e^{(1)} \otimes e^{(2)}\right) * \neq \alpha_{2}\left(e^{(1)} \otimes e^{(2)}\right) * \otimes \alpha_{1}\left(e^{(2)}\right)^{*}
$$

and $\mathscr{C}^{(\alpha)}$ is not any involutive cone. Proposition 4. 5b) yields now the assertion to be shown.

Remark. Noticing that $\mathscr{C}^{(\alpha)}$ is an alg-\# cone and using Corollary 4. 6, it follows that $\mathscr{C}^{(\alpha)}$ is also not any cone of $\tilde{\alpha}$-positivity.

The aim of the following is to define a $P$-functional $\phi$ on $\left(\mathbb{C}^{2}\right)_{\otimes}$. Let us start with the definition of a linear functional $T$ that will be used to define $\phi$. Let us put $T_{0}=1, T_{2 n-1}=0$. For defining $T_{2 n}$ let us consider subspaces 


$$
\begin{aligned}
Z_{2 n}= & \left\{g_{2 n}=e^{\left(i_{1}\right)} \otimes \cdots \otimes e^{\left(i_{2 n}\right)} \in X_{2 n} ; i_{1+j}=i_{2 n-J}, j=0,1, \ldots,\right. \\
& \left.n-1, i_{s} \in\{1,2\} \quad(s=1,2, \ldots, 2 n)\right\} .
\end{aligned}
$$

Let us define

$$
T_{2 n}\left(g_{2 n}\right)=\left\{\begin{array}{rll}
1 & \text { if } g_{2 n} \in Z_{2 n} \text { and } e^{\left(t_{n+1}\right)} \otimes \cdots \otimes e^{\left(i_{2 n}\right)} \in X_{n} \\
-1 & \text { if } \left.g_{2 n} \in Z_{2 n} \text { and } e^{\left(t_{n}+1\right.}\right) \otimes \cdots \otimes e^{\left(i_{2 n}\right)} \notin X_{n} \\
0 & \text { if } \quad g_{2 n} \in B_{2 n} \backslash Z_{2 n},
\end{array}\right.
$$

$n=1,2, \cdots$. Notice that, e.g.,

$$
\begin{aligned}
& T_{2}\left(e^{(1)} \otimes e^{(1)}\right)=-T_{2}\left(e^{(2)} \otimes e^{(2)}\right)=1, \\
& \begin{aligned}
T_{4}\left(e^{(1)} \otimes e^{(1)} \otimes e^{(1)} \otimes e^{(1)}\right) & =T_{4}\left(e^{(2)} \otimes e^{(2)} \otimes e^{(2)} \otimes e^{(2)}\right) \\
& =-T_{4}\left(e^{(1)} \otimes e^{(2)} \otimes e^{(2)} \otimes e^{(1)}\right)=1 .
\end{aligned}
\end{aligned}
$$

Setting $\mathscr{X}_{m}=\operatorname{span}\left(X_{m}\right), \mathscr{Y}_{m}=\operatorname{span}\left(B_{m} \backslash X_{m}\right)$, the (algebraic) direct decomposions

$$
\left(\mathbf{C}^{2}\right)_{m}=\mathscr{X}_{m} \oplus \mathscr{Y}_{m},
$$

$m=0,1,2, \ldots$, are implied.

Lemma 5. 4. The following hold for the linear functional $T=\left(T_{0}, 0, T_{2}, 0\right.$, $\left.T_{4}, \ldots\right)$.

i) $T_{2 n}\left(y_{2 n}\right)=0$ for $y_{2 n} \in \mathscr{Y}_{2 n}$,

ii) $T_{2 n}\left(\alpha_{n}\left(b_{n}\right) * \otimes b_{n}\right) \geqslant 0$ for $b_{n} \in B_{n}$,

iii) $\left|T_{n+m}\left(\alpha_{n}\left(g_{n}\right) * \otimes h_{m}\right)\right|^{2} \leqslant T_{2 n}\left(\alpha_{n}\left(g_{n}\right) * \otimes g_{n}\right) T_{2 m}\left(\alpha_{m}\left(h_{m}\right) * \otimes h_{m}\right)$, for $g_{n}$ $\in B_{n}, h_{m} \in B_{m}, n, m \in \mathbf{N}$.

Proof. i) follows immediately from (24). ii) Notice that $b_{n} \in B_{n}$ implies $b_{n}^{*} \otimes$ $b_{n} \in B_{2 n}$. Let us now distinguish the following: a) $b_{n}^{*} \otimes b_{n} \in B_{2 n} \backslash Z_{2 n}$, b) $b_{n}^{*} \otimes b_{n} \in$ $Z_{2 n}$ and $\left.b_{n} \in X_{n}, \mathrm{c}\right) b_{n}^{*} \otimes b_{n} \in Z_{2 n}$ and $b_{n} \notin X_{n}$. In the case of a), ii) holds because of $T_{2 n}\left(\alpha_{n}\left(b_{n}\right)^{*} \otimes b_{n}\right)=0$, where (23) was used. For b), ii) follows from

$$
T_{2 n}\left(\alpha_{n}\left(b_{n}\right) * \otimes b_{n}\right)=T_{2 n}\left(b_{n}^{*} \otimes b_{n}\right)=1,
$$

where (23), (24) were applied. In the case of c), ii) is analogously implied by

$$
T_{2 n}\left(\alpha_{n}\left(b_{n}\right) * \otimes b_{n}\right)=-T_{2 n}\left(b_{n}^{*} \otimes b_{n}\right)=-(-1)=1
$$

iii) If $n+m$ is odd or $n+m=2 l$ is even and $g_{n}^{*} \otimes h_{m} \notin Z_{2 l}$, then $T_{2 l}\left(\alpha_{n}\left(g_{n}\right) * \otimes h_{m}\right)$ 
$=0$. iii) is now a consequence of ii). Assume for the following that $g_{n}^{*} \otimes h_{m} \in Z_{2 l}$. Then,

$$
\left|T_{2 l}\left(\alpha_{n}\left(g_{n}\right) * \otimes h_{m}\right)\right|=1
$$

and $h_{m}^{*} \otimes h_{m} \in Z_{2 m}, g_{n}^{*} \otimes g_{n} \in Z_{2 n}$ follow. Arguing as in the proof of ii, b), c), it follows that

$$
T_{2 m}\left(\alpha_{m}\left(h_{m}\right) * \otimes h_{m}\right)=T_{2 n}\left(\alpha_{n}\left(g_{n}\right) * \otimes g_{n}\right)=1
$$

iii) is now a consequence of $(25)$ and $\left(25^{\prime}\right)$.

Let us consider the functional

$$
\phi=\frac{1}{2} T^{(\beta)},
$$

where $\left(\beta_{n}\right)_{n=0}^{\infty}$ is taken from (7) with $\omega_{i}=1, i=0,1,2, \ldots$. The following Proposition answers question $(Q)$ given in $\S 1$.

Proposition 5.5. a) $\phi$ is a P-functional on $\left(\mathbb{C}^{2}\right)_{\otimes}$, where the abstract conditional expectation is taken from Lemma 5.1 .

b) There are no $\pi_{\phi}$-invariant dual pairs.

c) $\phi$ is not the trivial extension of some $\alpha$-positive linear functional.

d) The Krein-space reconstructed from $\phi$ by GNS representation carries an indefinite metric $[x, y]=\phi\left(y^{*} x\right), x, y \in\left(\mathbb{C}^{2}\right)_{\otimes}$.

e) There is not any involution $\gamma_{1}: \mathbb{C}^{2} \longrightarrow \mathbb{C}^{2}$ such that $\gamma_{1}\left(f_{1}^{*}\right)=\gamma_{1}\left(f_{1}\right)^{*}, f_{1} \in$ $\mathbb{C}^{2}, T\left(\gamma\left(f^{*}\right) f\right) \geqslant 0, T(\gamma f)=T(f)$ for all $f \in\left(\mathbb{C}^{2}\right)_{\otimes}$, where $\gamma=\left(I_{0}, \gamma_{1}, \gamma_{2}, \ldots\right), \gamma_{n}=$ $\left(\gamma_{1}\right)^{\otimes n}(n=1,2, \ldots)$.

Proof. a), i) Noticing that $\phi_{0}=1,(11$, III $)$ is implied.

ii) Using Lemma 5.4 ii), iii), Lemma 2.5 implies that

$$
T^{(\beta)}\left(\alpha(f)^{*} f\right) \geqslant 0
$$

$f \in\left(\mathbb{C}^{2}\right)_{\otimes}$. Hence, $\phi$ satisfies $(11, \mathrm{II})$.

iii) Notice that $(I-P) x \in \mathscr{Y}$ for each $x \in\left(\mathbf{C}^{2}\right)_{\otimes}$, where $\mathscr{Y}=\bigoplus_{=1}^{\infty} \mathscr{Y}_{m}$. Applying Lemma 5. $4 \mathrm{i}), \phi(x-P(x))=0$ follows. Hence $(11$, II $)$ is implied.

iv) Consider $f_{2 m}=\sum \varphi_{j} b_{2 m}^{(j)}, \quad b_{2 m}^{(j)} \in B_{2 m}, \varphi_{j} \in \mathbf{C}$. Recalling (22) and (24), it follows that $T_{2 m}\left(b_{2 m}^{(j)} *\right)=T_{2 m}\left(b_{2 m}^{(j)}\right) \in \mathbb{R}$.

Hence, 


$$
T_{2 m}\left(f_{2 m}^{*}\right)=\sum_{J} \bar{\varphi}_{j} T_{2 m}\left(b_{2 m}^{(j)} *\right)=\sum_{J} \bar{\varphi}_{j} T_{2 m}\left(b_{2 m}^{(j)}\right)=\overline{T_{2 m}\left(f_{2 m}\right)}
$$

implying the Hermiticity of $\phi$.

b) is a consequence of Proposition 3.2 and $\left(24^{\prime}\right)$.

c) Assume that $\phi$ is the trivial extension of some functional $\Psi$. Because of dim $\left(E_{1}\right)=\operatorname{dim}\left(\mathbf{C}^{2}\right)=2$ and $(15), \ldots,\left(15^{\prime \prime}\right)$, it follows that $\Psi$ is defined on $\mathbf{C}_{\otimes}$. Hence, $\phi(x y)=\phi(y x)$ for all $x, y \in\left(\mathbf{C}^{2}\right)_{\otimes}$. Considering $x=e^{(1)}, y=e^{(2)} \otimes e^{(2)} \otimes e^{(1)}$, a contradiction follows from

$$
\begin{aligned}
& \phi(x y)=\phi_{4}\left(e^{(1)} \otimes e^{(2)} \otimes e^{(2)} \otimes e^{(1)}\right)=-1, \\
& \phi(y x)=\phi_{4}\left(e^{(2)} \otimes e^{(2)} \otimes e^{(1)} \otimes e^{(1)}\right)=0 .
\end{aligned}
$$

d) is obvious due to $\left(24^{\prime}\right)$.

e) Assume that there is an involution $\gamma_{1}$ satisfying the conditions stated in e). Write

$$
\gamma_{1}\left(e^{(j)}\right)=a_{j 1} e^{(1)}+a_{j 2} e^{(2)}
$$

where

$$
a_{11} a_{22}-a_{12} a_{21} \neq 0
$$

and $a_{j k} \in \mathbf{R}(j, k \in\{1,2\})$ due to $\gamma_{1}\left(f_{1}^{*}\right)=\gamma_{1}\left(f_{1}\right)^{*}, f_{1} \in \mathbf{C}^{2}$. Then,

$$
\begin{aligned}
0 & =T_{2}\left(\gamma_{1}\left(e^{(2)}\right) \otimes \gamma_{1}\left(e^{(2)}\right)-T_{2}\left(e^{(2)} \otimes e^{(2)}\right)=\left(a_{21}\right)^{2} T_{2}\left(e^{(1)} \otimes e^{(1)}\right)\right. \\
& =\left(a_{21}\right)^{2}, \\
0 & \leq T_{2}\left(\gamma_{1}\left(e^{(1)} \otimes e^{(1)}\right)=a_{11},\right.
\end{aligned}
$$

and (27) imply $a_{21}=0, a_{11}>0, a_{22} \neq 0$. Now,

$$
\begin{aligned}
0 \leq & T_{6}\left(\gamma_{1}\left(e^{(2)}\right) \otimes\left(\gamma_{1}\left(e^{(1)}\right) \otimes \gamma_{1}\left(e^{(2)}\right) \otimes e^{(2)} \otimes e^{(1)} \otimes e^{(2)}\right)\right. \\
& =a_{11}\left(a_{22}\right)^{2} T_{6}\left(e^{(2)} \otimes e^{(1)} \otimes e^{(2)} \otimes e^{(2)} \otimes e^{(1)} \otimes e^{(2)}\right)=-a_{11}\left(a_{22}\right)^{2}
\end{aligned}
$$

yield a contradiction.

Remark 5. 6. a) Proposition 5. 4c), e) shows that there is not any cone of $\alpha$ positivity in $\left(\mathbf{C}^{2}\right)_{\otimes}$ such that $\phi$ is invariant and also positive with respect to that cone. Hence, it is not possible to apply the method of $\alpha$-positivity for constructing 
a Krein space theory.

b) The proof of Proposition 5. 2 shows that every sequence $\left\{X_{n}\right\}_{n=0}^{\infty}$ of sets $X_{n}$ $\subset E_{n}$ defines an abstract conditional expectation $P$ on a tensor algebra $E_{\otimes}$ if

i) $X_{0}=\{1\}$,

ii) $X_{n}=X_{n}^{*}, n=1,2, \ldots$,

iii) Lemma 5. 1a) applies

are satisfied. Along these lines, there is the following straightforward generalization of the definitions given in (20). Let us be given a vector space $E$ with $\operatorname{dim}(E)=\infty$ and an involution "**. Choose then a sequence $\left(e^{(t)}\right)_{l=1}^{\infty}$ of linearly independent elements $e^{(i)}=e^{(i)} * \in E$. Analogously to (20) define sets $X_{n} \subset E_{n}$ by

$$
\begin{aligned}
X_{n}= & \left\{e^{\left(i_{1}\right)} \otimes \cdots \otimes e^{\left(i_{n}\right)} ; i_{j} \in \mathbb{N}(j=1,2, \ldots, n), \text { each } e^{(l)}\right. \\
& \text { occurs only in a cluster of } \left.i \text { neighboring elements } e^{(i)}\right\} .
\end{aligned}
$$

Substitute $\left(e^{(i)}\right)_{i=1}^{\infty}$ to a basis $B_{1}=\left\{e^{(\delta)}\right\}_{\delta \in A}$ with $e^{(\delta)}=e^{(\delta)} * \in E, \delta \in A$ (A is a set of indices). Noticing that

$$
B_{n}=\left\{e^{\left(i_{1}\right)} \otimes \cdots \otimes e^{\left(i_{n}\right)} ; i_{j} \in A(j=1,2, \ldots, n)\right\}
$$

is a basis of $E_{n}$ define projections $P_{n}$ analogously to (21). It follows now that $P=$ $\left(I_{0}, P_{1}, P_{2}, \ldots\right)$ represents an abstract conditional expectation on $E_{\otimes}$.

c) Analyzing the construction of the functional $\phi$, it follows that every sequence $\left\{X_{n}\right\}_{n=0}^{\infty}, X_{n} \subset E_{n}$, defines a $P$-functional by (24), and (26), if $\left\{X_{n}\right\}_{n=0}^{\infty}$ satisfies bi), ..., biii) and Lemma 5. 1b) applies in addition. Thus a further example of a $P$-functional $\tilde{\phi}$ on $\left(\mathbb{C}^{2}\right)_{\otimes}$ is given in the following way. Let $X_{n}$ be the set of all the elements $g_{n}=e^{\left(i_{1}\right)} \otimes \ldots \otimes e^{\left(i_{n}\right)} \in E_{n}, i_{j} \in\{1,2\}(j=1,2, \ldots, n)$, such that $z_{s}\left(g_{n}\right)$ is even or 0 for $s=1,2$, where $z_{s}\left(g_{n}\right)$ stands for the number of factors $e^{(s)}$ occuring in $g_{n}$. It follows straightforwardly that an analogous statement as the one of Proposition 5. 5e) also applies to $\tilde{\phi}$.

\section{Acknowledgements}

The author is indepted to Professor S. Ôta for drawing his attention to the problem and for helpful discussions. The inquiries of the referee are gratefully acknowledged.

\section{References}

[1] Antoine, J.-P. and Ota, S., Unbounded GNS representations of a *-algebra in a Krein space, Lett. Math. Phys., 18 (1989), 267-274.

[2] Araki, H., On a pathology in indefinite inner product space, Comm. Math. Phys., 85 (1982), 121- 
128.

[3] Araki, H., Indecomposable representations with invariant inner product. A theory of the Gupta-Bleuler triplet, Comm. Math. Phys., 97 (1985), 149-159.

[4] Asisov, T. J. and Iochvidov, I. S., Foundations of the theory of linear operators in spaces with indefinite metric, Moscow 1986, Nauka (in Russian).

[ 5 ] Bognar, J., Indefinite inner product spaces, Springer-Verlag, Berlin 1974.

[6] Bolgoljubov, N. N., Logunov, A. A., Oksak, A. I., Todorov, I. T., Common principles of quantum field theory, Moscow 1987, Nauka (in Russian).

[ 7 ] Bongaarts, P. J. M., Maxwell's equations in axiomatic quantum field theory, I. Field tensor and potentials, J. Math. Phys., 18 (1977) 1510-1516; II. Covariant and non-covariant gauges, J. Math. Phys., 23 (1982), 1881-1898.

[8] Borchers, H. J., On the structure of the algebra of field operators, Nuovo Cimento, 24 (1962), 214-236; Algebraic aspects of Wightman field theory, in Sen R. N. and Weil, C. (eds.), Statistical Mechanics and Field Theory, Wiley, New York, 1972.

[9] Dadashyan, K. Yu. and Khoruzhii, S. S., On field algebras in quantum theory with indefinite metric, Teor. Mat. Fiz., 54 (1983), 57-77.

[10] Dubin, D. A. and Tarski, J., Indefinite metric resulting from regularization in the infrared region, J. Math. Phys., 7 (1966), 574-577.

[11] Grundling, H., Systems with outer constraints. Gupta-Bleuler electromagnetism as an algebraic field theory, Comm. Math. Phys., 114 (1988), 69-91.

[12] Hofmann, G., On the closed hull of subsets of inductive limits, Publ. RIMS, Kyoto Univ., 27 (1991), 813-826.

[13] - On algebraic \#-cones in topological tensor-algebras, I. Basic properties and normality, Publ. RIMS, Kyoto Univ., 28 (1992), 455-494.

[14] - An explicite realization of a GNS representation in a Krein space, Publ. RIMS, Kyoto Univ., 29 (1993), 267-287.

[15] Ito, K. R., Canonical linear transformation on Fock space with an indefinite metric, Publ. RIMS, Kyoto Univ., 14 (1978), 503-556.

[16] Jadczyk, A., Geometry of indefinite-metric spaces, Rep. Math. Phys., 2 (1971), 263-276.

[17] Jakóbczyk, L., Borchers algebra formulation of an indefinite inner product quantum field theory, J. Math. Phys., 25 (1984), 617-622.

[18] Canonical quantization with indefinite inner product, Ann. of Phys., $16 \mathbb{1}$ (1985), 314-336.

[19] Jakóbczyk, L. and Strocchi, F., Krein Structures for Wightman and Schwinger functions, $J$. Math. Phys., 29 (1988), 1231-1235.

[20] - Euclidean formulation of quantum field theory without positivity, Comm. Math. Phys., 119 (1988), 529-541.

[21] Kugo, T. and Ojima, I., Local covariant operator formalism of non-abelian gauge theories and quark confinement problem, Suppl. Progr. Theor. Phys., 66 (1979), 1-130.

[22] Morchio, G. and Strocchi, F., Infrared problems, Higgs phenomena and long range interaction, in Velo, G. and Wightman, A. S. (eds.), Fundamental Problems of Gauge Field Theory (Proc. Erice 1985), Plenum, New York, 1986.

[23] - Infrared singularities, vacuum structure and pure phases in local quantum field theory, Ann. Inst. H. Poincaré, 33 (1989), 251-282.

[24] Nakanishi, N., Indefinite-metric quantum field theory of general relativity, Progr. Theor. Phys., 59 (1978), 972-984.

[25] Ôta, S., Unbounded representations of a *-algebra on indefinite metric space, Ann. Inst. $H$. 
Poincaré, 48 (1988), 333-353.

[26] Sakai, S., $C^{*}$ Algebras and $W^{*}$ Algebras, Springer-Verlag, Berlin, 1970.

[27] Schmüdgen, K., Unbounded Operator Algebras and Representation Theory, Akademie-Verlag, Berlin 1990.

[28] Strocchi, F., Spontaneous symmetry breaking in local gauge quantum field theory ; the Higgs mechanism, Comm. Math. Phys., 56 (1977), 57-78.

[29] Strocchi, F. and Wightman, A. S., Proof of charge selection rule in local relativistic quantum field theory, J. Math. Phys., 15 (1974), 2198-2224.

[30] Uhlmann, A., Über die Definition der Quantenfelder nach Wightman und Haag, Wiss. Z. Univ. Leipzig, 11 (1962), 213-217.

[31] Völkel, A. H., Unbounded representations of symmetry groups in gauge quantum field theory. I \& II, J. Math. Phys., 26 (1985), 2956-2973; and 27 (1986), 1113-1127.

[32] Yngvason, J., On the algebra of test functions for field operators, Comm. Math. Phys., 34 (1973), 315-333.

[33] Remarks on the reconstruction theorem for field theories with indefinite metric, Rep. Math. Phys., 12 (1977), 57-64. 